\title{
Physical and chemical characterisation of some silicas and silica derivatives
}

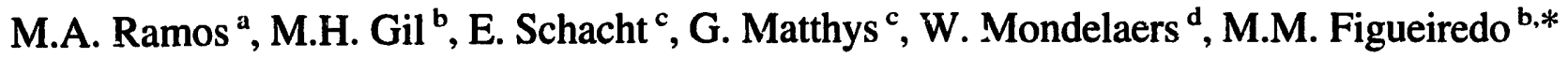 \\ “Instituto Superior de Engenharia, Inst. Politécnico de Coimbra, 3000 Coimbra, Portugal \\ ' Departamento de Engenharia Química, Universidade de Coimbra, Largo Marquês de Pombal, 3000 Coimbra. Portıga! \\ 'Polvmer Materials Research Group, University of Ghent, Ghent, Belgium \\ ¿Department of Irradiation Physics, University of Ghent, Ghent, Belgium
}

Reccived 12 February 1998; received in revised form 13 April 1998; accepted 14 April 1998

\begin{abstract}
An extensive study of four types of porous silica supports has been performed, with particular emphasis on their physical and morphological characteristics. These silicas were modified by reacting the surface silanol groups present with either 3-(trimethoxysilyl)propylmethacrylate or 3-giycidoxypropyltrimethoxysilane or 3-aminopropyltriethoxysilane, to produce derivatives with suitable functional groups for further utilisation in the immobilisation of biological compounds. The silicas and their derivatives used were fully characterised with regard to particle size distribution ( laser light scattering), specific surface area (BET method), pore size distribution ( gas adsorption and mercury porosineetry), density (helium pycnometry), yield of grafting (TGA) and chemical composition (FTIR/DRIFT).

(C) 1998 Elsevier Science S.A. All rights reserved.
\end{abstract}

Keywords: Silica; Silanisation; Particle size; Surface area; Pore size distribution

\section{Introduction}

Silica derivatives are among the most common packing materials used in chromatographic columns (partitioning chromatography, hydrophobic and affinity separations), as well as in reactors operating with immobilised biological compounds. The main reasons for this choice depend on the mechanical and physical characteristics of the silicas, in particular their hardness, non-compressibility and high controlled surface area, which make them specially suitable for packed beds.

The chemical derivatisation of the silica particles, which enables covalent linkage of various compounds (either biological or not), leads to considerable changes in their physical and chemical properties. Indeed, specific silica carriers for immobilisation reactions can be produced by reacting the silica hydroxyl groups with selected silane compounds. As the reactions take place at the support surfaces, the knowledge of the texture of the carrier is of crucial importance. It has been recognised $[1-9]$ that the particle surface area, and, specially, the pore size distribution, greatly control the final behaviour of the supports. Additionally, besides providing higher surface areas, small diameter pores may limit biolog-

* Corresponding author. Tel.: + 351-39-828392; Fax: + 351-39-841138 ical compound immobilisation as well as diffusion of the substrates and reaction products. Therefore, an extensive knowledge of the support properties is essential for better understanding of the carrier performances.

The emphasis of the present work is on the characterisation of four different silicas, which were further modified by reaction with silane compounds to change their surface chemistry. For this purpose, new functional groups suitable for the fixation of biological compounds were introduced. The materials obtained can be used either for chromatographic separations or as bioreactors. The original silicas were characterised, basically, with respect to their morphological properties (i.e., particle size, surface area and pore size distribution), whereas for the silica derivatives, a somewhat different strategy was undertaken. With the silanised samples the intention was not only to examine the extent of grafting but also to investigate the consequences with regard to the support surface texture.

\section{Experimental}

\section{I. Materials}

The four porous silicas utilised in this work were supplied by Macherey-Nagel (Düren, Germany). Table 1 summarises 
Table 1

Data supplied by the manufacturer for the original silicas

\begin{tabular}{lllll}
\hline $\begin{array}{l}\text { Supplier } \\
\text { identification }\end{array}$ & $\begin{array}{l}\text { Abbreviation } \\
\text { used }\end{array}$ & $\begin{array}{l}\text { Pore } \\
\text { diameter } \\
(\AA)\end{array}$ & $\begin{array}{l}\text { Surface } \\
(\mathrm{BET}) \\
\left(\mathrm{m}^{2} \mathrm{~g}^{-1}\right)\end{array}$ & $\begin{array}{l}\text { Density } \\
\left(\mathrm{g} \mathrm{ml}^{-1}\right)\end{array}$ \\
\hline Polygosil 60 & S1 & 60 & 450 & 0.45 \\
Nucleosil 100 & $\mathrm{S} 2$ & 100 & 350 & 0.36 \\
Nucleosil 300 & S3 & 300 & 100 & 6.45 \\
Nucleosil 1000 & $\mathrm{S} 4$ & 1000 & 25 & 0.45 \\
\hline
\end{tabular}

the specifications given by the manufacturer. However, no information was provided regarding the techr iques used to measure vore size and density. In addition, it was mentioned that the particle size, for all the samples, ranged from 25 to $40 \mu \mathrm{m}$, and also that Polygosil was irregular in shape whereas Nucleosil particles were nearly spherical. Therefore, a more detailed characterization was initiated.

For silanisation, the following systems were used: 3(trimethoxysilyl)propyl methacrylate, 3-glycidoxypropyltrimethoxysilane and 3-aninopropyltriethoxysilane, respectively abbreviated as Sa, Sb and Sc. These compounds were purchased from Aldrich (Bornem, Belgium). Although all these reagents were used to obtain supports suitable for biological immobilisations, the first was especially selected to graft monomers with new functional groups (e.g.. $-\mathrm{OH}$ and $-\mathrm{COOH}$ ).

\subsection{Functionalisation of the silica}

The silicas were dried at $100^{\circ} \mathrm{C}$ overnight and subsequently suspended in toluene $(20 \%, w / v)$ under nitrogen atmosphere. The samples SI and S2 (Table I) were treated in a $10 \%(\mathrm{v} / \mathrm{v})$ solution of silane compound/toluene whereas for the samples S3 and S4 this percentage was reduced to $5 \%$. These suspensions were refluxed for $2 h$. The functionalised silicas were allowed to cool to room temperature. filtered. washed three times with toluene, rinsed with methanol and finally dried at $60^{\circ} \mathrm{C}$ overnight.

\subsection{Assesssment of grafted material}

The amount of grafted material in the samples was determined by weight loss in a TGA apparatus (2950 TGA from Polymer Laboratories Thermogravimetric Analyser), over the range 25 to $700^{\circ} \mathrm{C}$, with a heating rate of $20^{\circ} \mathrm{C} / \mathrm{min}$. using air as the purge gas. To eliminate error sources that could arise from the presence of residual methanol, water or toluene. the weight loss was only considered for temperatures above $130^{\circ} \mathrm{C}$.

The percentage of the grafted silane was calculated using:

$\%$ of grafted silane $=100\left(w_{i}-w_{\mathrm{f}}\right) / w_{\mathrm{f}}$

where $w_{1}$ and $w_{\mathrm{f}}$ are the initial and the final weight of the samples. respectively, the latter taken as the weight of the original silica present in the sample.

\subsection{Microscopy}

Samples were also visualised with an optical microscope (Olympus-BH2) coupled to an image analysis system. However, the main objective was to examine the shape of the particles and the state of agglomeration, rather than to determine their particle size distribution. A more expeditious way was used to obtain the latter, as described below.

\subsection{Particle size analysis}

The particle size distributions of all the samples were obtained by laser light scattering in the LS 130 from Coulter Electronics, using water as the suspending medium. The Fraunhofer theory [10] was used to deconvolute the corresponding scattering patterns, after ensuring that the application of Mie theory [10], using the refractive index of silica (1.46), generated coincident size distributions.

\subsection{Density méasurements}

The true densities of the samples, or better. their true volume, were determined by gas ( helium ) pycnometry, utilising an Accupyc 1330 apparatus from Micromeritics. An equilibration rate of $0.0050 \mathrm{psig} / \mathrm{min}$ was used and the results were the average of at least three runs.

\subsection{Surface area determinutions}

The surface area measurements were performed bv gas adsorption in the ASAP ( Accelerated Surface Area and Porosimetry) 2000 from Micromeritics. using nitrogen as the adsorptive. The samples were previously degassed to below $50 \mu \mathrm{m} \mathrm{Hg}$ at room temperature and analyses were performed at $77 \mathrm{~K}$. using liquid nitrogen. The equilibration interval was $5 \mathrm{~s}$. The surface area was calculated using the BET method [III. Pore volume and area distributions, based on the BJH calculation [12], were evaluated by the apparatus software.

\subsection{Mercury porosimetry}

A Micromeritics Poresizer 9320 Mercury Porosimeter was utilised for the mercury intrusion/extrusion experiments. All the samples were degassed before analysis at a vacuum pressure below $50 \mu \mathrm{m} \mathrm{Hg}$. This equipment allows both low pressure (up to 25 psia) and high pressure (up to 30000 psia) measurements.

By knowing the volume intruded at 25 psia, known as the bulk volume ( since it includes solid samplc, pores and interstices), and the material weight, it is possible to calculate the bulk density. High pressure runs were made with an equilibration time of $20 \mathrm{~s}$ and a maximum intrusion volume of $0.0500 \mathrm{ml} / \mathrm{g}$.

The porograms ( intruded or extruded volumes versus pressure) obtained were converted into pore diameter distribution 
rurves (cumulative and differential) according to the Washburn equation [13].

\subsection{Water vapour sorption capacity}

The samples were placed in a container with a saturated solution of copper sulphate ( $98 \% \mathrm{RH}$ ) and kept at $25^{\circ} \mathrm{C}$ Littl constant weight. The samples were subsequently weighed every minute for $10 \mathrm{~min}$. The initial sorption capac:ty was obtained from plots after extrapolation to zero time. Each sample was dried to constant mass in a dryer at $105^{\circ} \mathrm{C}$ for 6 $h$. The percentage of water uptake was given by

$\%$ sorption $=100\left(M_{1}-M_{1}\right) / M_{1}$

where $M_{1}$ is the mass at zero time and $M_{1}$ is the final dry mass.

\section{Results and discussion}

\subsection{Particle shape and sise distribution}

Fig. 1 shows the micrographs of Polygosil (S1) and of Nucleosil (S3). As it can be seen, the Polygosil particles are irregular, whereas those of Nucleosil are approximately spherical. Although only the S3 sample is shown, identical shapes (i.e., nearly spherical) were found for the particles of the remaining Nucleosil samples. Nevertheless, from microscope observations, it was possible to notice that some of the Nucleosil particles consisted of agglomerates, that could be a result of the nucleation manufacture process. These agglomerates are easily dispersed by agitation, producing smaller primary particles, generally irregular in shape.

The volume weighed particle size distributions are shown in Fig. 2, for Polygosil and for one of the Nucleosil samples (S4), and are quantified in Table 2, for all samples, in terms of $d_{10}, d_{50}$ (median size) and $d_{90}$, corresponding, respectively, to cumulative volume frequencies of $10 \%, 50 \%$ and 90\%.
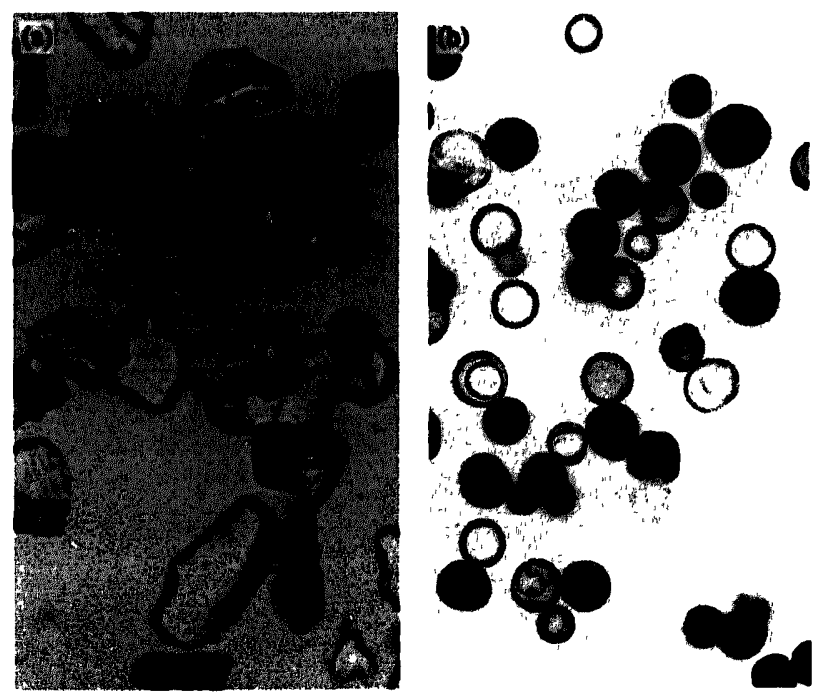

Fig. 1. Micrographs (240X) of Polygosil (a) and Nucleosil S3 (b).

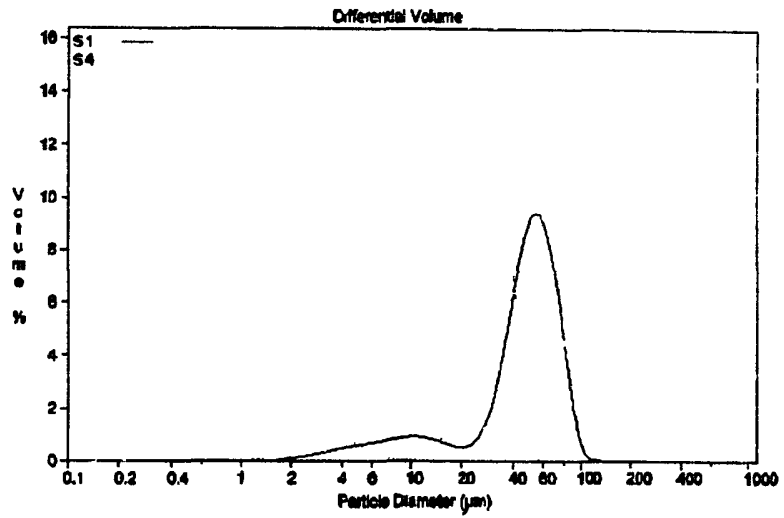

Fig. 2. Differential particle size distribution of Polygosil ( $\mathrm{S}$ ) and of one Nucleosil sample (S4), determined by laser diffraction.

Tuble 2

Particle size distribution of the various silicas in terms of $d_{10,}, d_{9 t}$ and $d_{141}$ $(\mu \mathrm{m})$, determined by laser diffraction

\begin{tabular}{lrll}
\hline Sample & $d_{11}$ & $d_{511}$ & $d_{111}$ \\
\hline S1 & 10.30 & 48.54 & 74.78 \\
S2 & 9.06 & 32.74 & 42.46 \\
S3 & 11.36 & 30.22 & 40.64 \\
S4 & 11.84 & 29.33 & 39.16 \\
\hline
\end{tabular}

The results obtained indicate that the Polygosil sample. besides presenting a larger size (clearly noticeable by microscopy), exhibits a broader size distribution than the Nucleosil samples, which are all quite similar. Moreover, the size distribution curves always show a bimodal distribution, the smallest peak comprising particles below 20 or $10 \mu \mathrm{m}$, depending on the sample (the largest value corresponding to Polygosil).

\subsection{Chemical characte risation of the silanised silicas}

As referred to earlier, the silicas were modified by silanisation according the reactions indicated in Fig. 3. The yield of grafting, evaluated by TGA, is indicated in Table 3 and will be discussed later. In this table, the silanised samples are designated by $\mathbf{S} x \boldsymbol{S} y$, where $\mathbf{S} x$ denotes the original silica ( $\mathrm{S}$. S2, S3 or S4) and Sy specifies the silane compound used ( $\mathrm{Sa}$, $\mathrm{Sb}$ or $\mathrm{Sc}$ ).

The original silica, as well as the grafted silicas were characterised by FTIR/DRIFT using a Nicolet Magna IR ${ }^{\text {NiD }}$ Spectrometer 750. The FTIR/DRIFT spectrum $\left(\mathrm{cm}^{-1}\right)$ is the

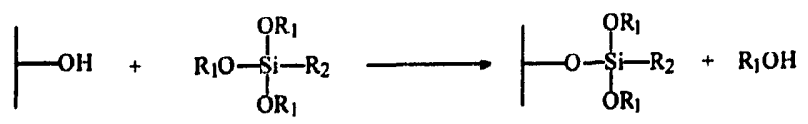

$\mathrm{R}_{1}=\mathrm{CH}_{3}$ or $\mathrm{C}_{2} \mathrm{H}_{5}$

$\mathrm{R}_{2}=\left(\mathrm{CH}_{2}\right)_{3} \mathrm{OOCClam}_{\mathrm{CH}_{3}}$ or $\left(\mathrm{CH}_{2}\right)_{3} \mathrm{OCH}_{2} \mathrm{CH}_{\mathrm{H}} \mathrm{CH}_{2}$ or $\left(\mathrm{CH}_{2}\right)_{3} \mathrm{NH}_{2}$

Fig. 3. Silanisation reactions of the silica. 
Table 3

Characterisation of the original silicas and their derivatives

\begin{tabular}{|c|c|c|c|c|c|c|c|c|}
\hline Sample & $\begin{array}{l}\text { Surface } \\
\text { area } \\
\left(\mathrm{m}^{2} \mathrm{~g}^{-1}\right)\end{array}$ & $\begin{array}{l}\text { Mean pore } \\
\text { diameter } \\
(\AA)\end{array}$ & $\begin{array}{l}\text { Mean pore } \\
\text { diameter }^{\mathrm{d}} \\
(\dot{A})\end{array}$ & $\begin{array}{l}\text { True } \\
\text { density } \\
\left(\mathrm{g} \mathrm{ml}^{-1}\right)\end{array}$ & $\begin{array}{l}\text { Bulk } \\
\text { density } \\
\left(\mathrm{g} \mathrm{ml}^{-1}\right)\end{array}$ & $\begin{array}{l}\text { Porosity }{ }^{z} \\
(\%)\end{array}$ & $\begin{array}{l}\text { Yield of } \\
\text { grafting } \\
(\%)\end{array}$ & $\begin{array}{l}\text { Water vapour } \\
\text { sorption } \\
(\%)\end{array}$ \\
\hline SI & 468 & $47^{\circ}$ & 127 & 2.21 & 0.45 & 79.6 & - & 80.6 \\
\hline SISa & 391 & $41^{\mathrm{b}}$ & - & 1.92 & - & - & 11.8 & 55.8 \\
\hline SISb & 383 & $43^{n}$ & - & 2.02 & - & - & 10.2 & 60.2 \\
\hline SISc & 363 & $42^{\circ}$ & - & 2.05 & - & - & 8.5 & 57.3 \\
\hline S2 & 318 & $117^{b}$ & 121 & 2.16 & 0.34 & 84.3 & - & 69.0 \\
\hline S2Sa & 276 & $115^{h}$ & - & 2.02 & - & - & 8.3 & 54.4 \\
\hline S2Sh & 266 & $108^{h}$ & - & 2.05 & - & - & 7.6 & 53.5 \\
\hline S3 & 61 & $130 / 550^{\circ}$ & 316 & 2.27 & 0.43 & 81.1 & - & 24.4 \\
\hline S3Sa & 56 & - & - & 2.26 & - & - & 2.3 & - \\
\hline $\mathrm{S3Sb}$ & 57 & - & - & - & - & - & 2.8 & 15.1 \\
\hline S3Se & 58 & - & - & - & - & - & 1.5 & 21.9 \\
\hline 54 & 48 & $130 / 1200^{c}$ & 464 & 2.34 & 0.39 & 83.3 & - & 11.3 \\
\hline S4Su & 41 & - & - & 2.29 & - & - & 0.4 & 9.7 \\
\hline $\mathrm{SASb}$ & 41 & - & - & - & - & - & 0.2 & 9.3 \\
\hline S4Sc & 40 & - & - & - & - & - & 1.2 & 6.9 \\
\hline
\end{tabular}

"Evaluated from BET.

$B=4 V / A$, where $V$ is the total pore volume and $A$ the corresponding surface area from BJH desorption branch.

'Mode of first peak/mode of second peak, determined by mercury porosimetry.

$=4 V / A$, where $V$ is the corrected total intruded volume and $A$ the corresponding surface area.

- Determined by helium pycnometry.

' Determined by mercury porosimetry (at 25 psia).

$*$ Calculated as ( (true density-bulk density)/(rue density) $\times 100$.

" Determined by TGA.

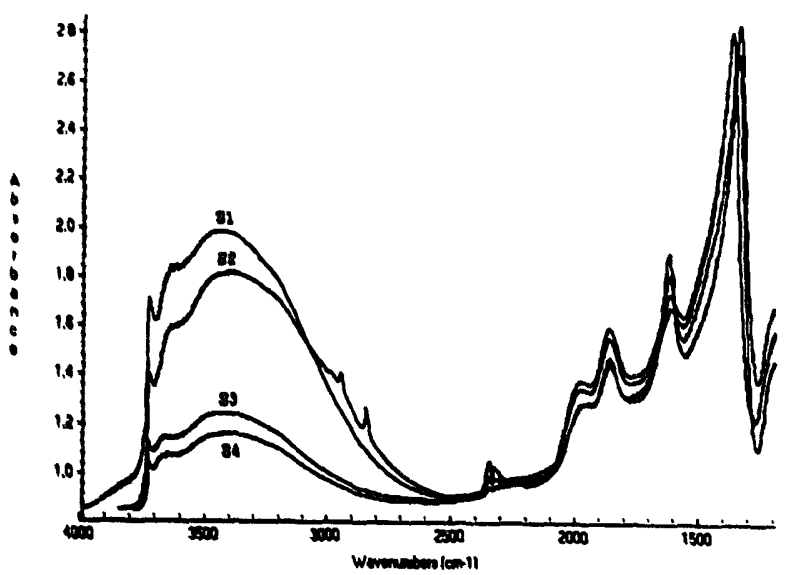

Fig, 4. FTIR/DRIFT spectra for the original silica samples (S1, S2. S3 and St).

same as that of the normal silica [14], with the characteristic 810 (Si-O-Si silica), 1110 ( Si-O-Si silica) and 3400 (O-H silica) $\mathrm{cm}^{-1}$ bands. However, the spectra of the four original silicas, presented in Fig. 4, indicate that these samples exhibit considerable differences in the percentage of $-\mathrm{OH}$ groups.

The spectra of the S1 sample after silanisation with different compounds are shown in Fig. 5. In this case, all derivatised samples show bands at $2950\left(-\mathrm{CH}_{2}\right.$ and $\left.-\mathrm{CH}_{3}\right)$ and 1460 $\left(-\mathrm{CH}_{2}\right.$ and $\left.-\mathrm{CH}_{3}\right) \mathrm{cm}^{-1}$. In addition, for $\mathrm{SISa}$ sample there is a band at 1700 ( $>C=0$, aliphatic ester $)$, and for S1Sc a

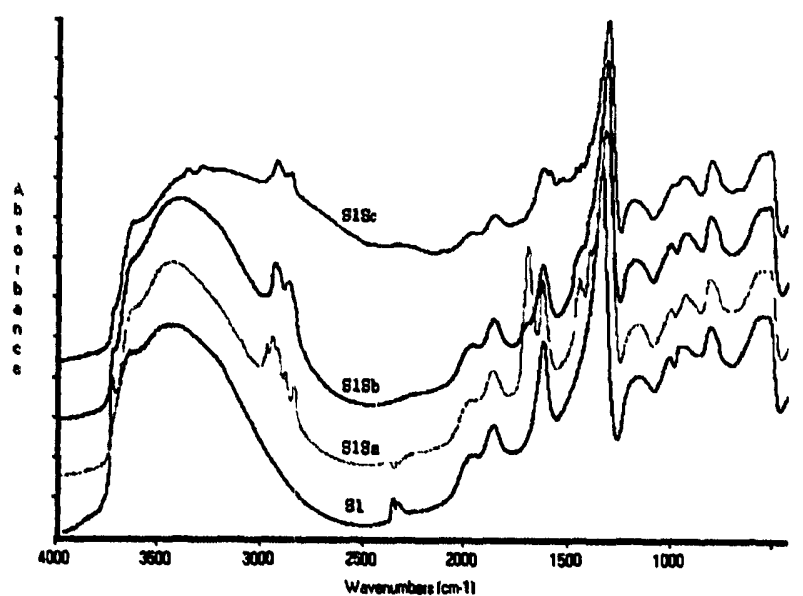

Fig. 5. FTIR/DRIFT spectra for the original silica $S 1$ and the corresponding silanised derivatives (SISa.SISb and SISc).

band at 1650 (- $\mathrm{NH}_{2}$, amino group ) and a broad band at 3330 3380 ( $-\mathrm{NH}_{2}$, amino group) $\mathrm{cm}^{-1}$.

\subsection{Surface area, porosity and density}

The isotherms obtained for all silicas were identical, a typical example being shown in Fig. 6. Their shapes are similar to those of type IV, denoting the presence of mesopores, with hysteresis between type $\mathrm{H} 1$ and $\mathrm{H} 2[15,16]$. The BET surface areas were calculated from the corresponding plots with correlation factors higher than 0.9995 and $C$ values 


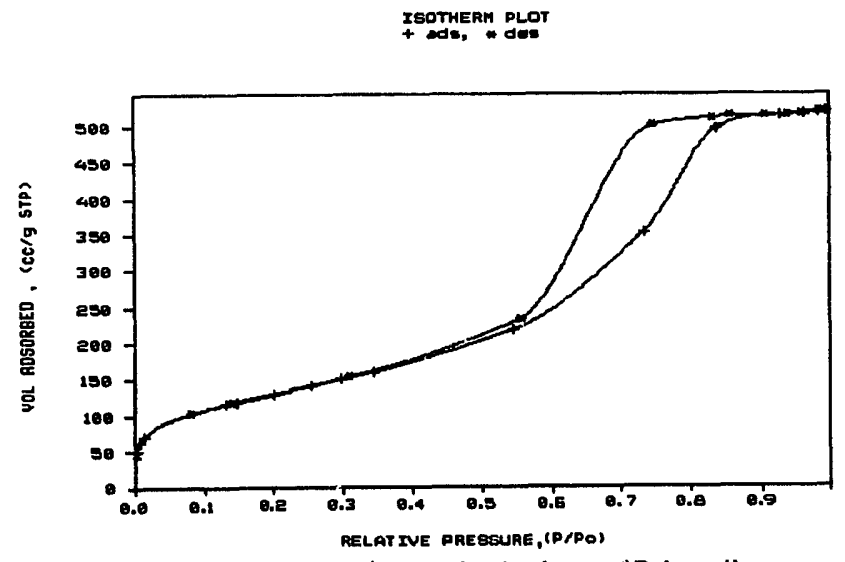

Fig. 6. Gas adsorption/desorption isotherm of Polygosil.

ranging from 39 to 288 , demonstrating the validity of this method [16]. The specific surface areas obtained for both the original silicas and their derivatives are listed in Table 3.

Although the knowledge of the sample surface area is important, as already pointed out, the pore size distribution is even more critical, since it greatly affects the yield of the biological immobilisation as well as the diffusion controlled phenomena. Since the gas adsorption technique is not very accurate with respect to pore size distribution in the range of pressures near saturation, it was decided to use additionally, mercury porosimetry. Further, the pore size range is not the same in both techniques, the latter being more adequate for measuring larger pores. A typical porogram is shown in Fig. 7. The stepped intrusion curve clearly denotes different pore size ranges. Furthermore, it can be noticed that a considerable volume was intruded at relative low pressures ( corresponding to pores larger than $30000 \AA$ ), which is most certainly related to the filling of the large interparticle voids. This volume was thus excluded from the calculations of the particle pore volume and area.

The computation of the pore size distribution, from gas adsorption, was based on the BJH method using the desorp-

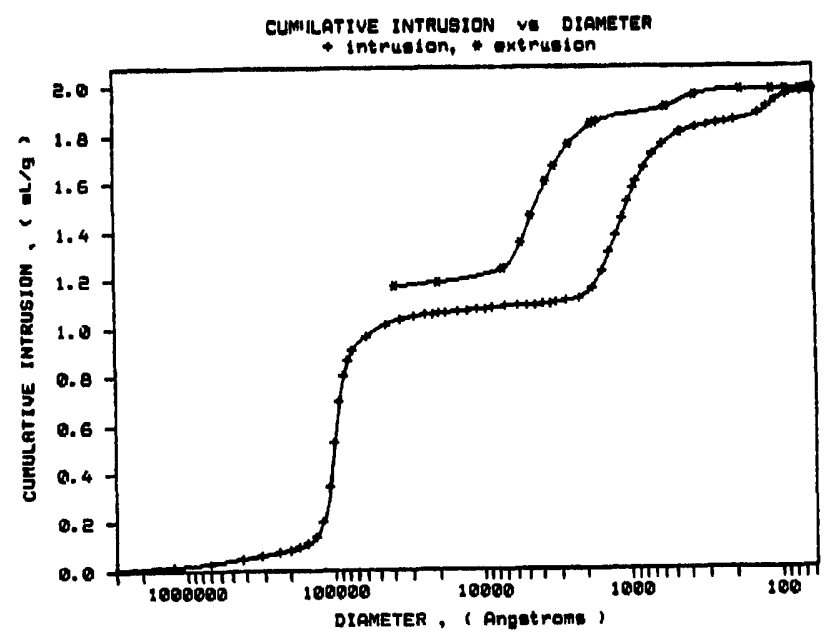

Fig. 7. Mercury intrusion/extrusion curve of one Nucleosil sample (S4). tion branch, which appears to be favoured by most workers, both because it corresponds to a more stable adsorbents condition and it correlates better with the mercury porosimetry data $[16,17]$.

Fig. 8 shows the pore size distribution of the primary silicas on an incremental (derivative) basis, $\mathrm{d} V / \mathrm{d} \log (D)$, to highlight the differences between the supports. From these plots, the following conclusions can be withdrawn.

(i) Samples S1 and S2 exhibit a unimodal distribution of pores, whose sizes are well within the size range measured by gas adsorption but are near the lower limit of detection of the porosimeter. The calculation of the mean pore size will therefore be more accurate when derived from gas adsorption than from mercury porosimetry. These values are presented in the second and third columns of Table 3. As expected, for sample $\mathbf{S} 1$ (which contains smaller pores), the mean pore size $(4 V / A)$ given by porosimetry is clearly overestimated (127 $\AA$ for porosimetry against $47 \AA$ for gas adsorption). This is mainly due to the inaccuracy of the area measured by this technique, which does not take into account values corresponding to pores smaller than $60 \AA$. For sample S2, the results obtained by both techniques are in good agreement ( $121 \AA$ for porosimetry and $117 \AA$ for gas adsorption).

(ii) With regard to samples $\mathrm{S} 3$ and $\mathrm{S} 4$, a bimodal distribution of pore sizes was detected by both techniques. In this case, the mean pore diameter (third column of Table 3 ) lacks physical meaning, and it is better to present instead the modes of the two peaks ( second column of Table 3). Further, the values listed are those of porosimetry, which, as mentioned, is more reliable for sizing larger pores. Both samples show one peak at $130 \AA$, and a second at $550 \AA$ for sample $S 3$ and at $1200 \AA$ for sample S4.

The isotherms of the silica derivatives are similar to those corresponding to the non-silanised samples (data not shown). However, the hysteresis loops of the former are always slightly deviated to the left, indicating that grafting decreases pore diameters. This is confirmed by the lower values reported in Table 3 for both surface area and mean pore diameter of the silanised samples.

Table 3 also includes the values of the bulk density and porosity of the original silicas and the true density of most of the samples. The first two parameters are useful to evaluate the packing characteristics of the supports. The true density of the modified silicas was found to decrease with the silanisation reaction, which can be explained by the larger increase in the volume (due to grafting) than the weight of the sample.

\subsection{Yield of grafting}

From the results presented in Table 3 concerning the percentage of grafted silane, it can be noticed that the higher the surface area of the original silica, the higher the yield of grafting. This was predictable since the silica reactivity (i.e., the concentration of hydroxyl groups available) is directly proportional to the surface area. 

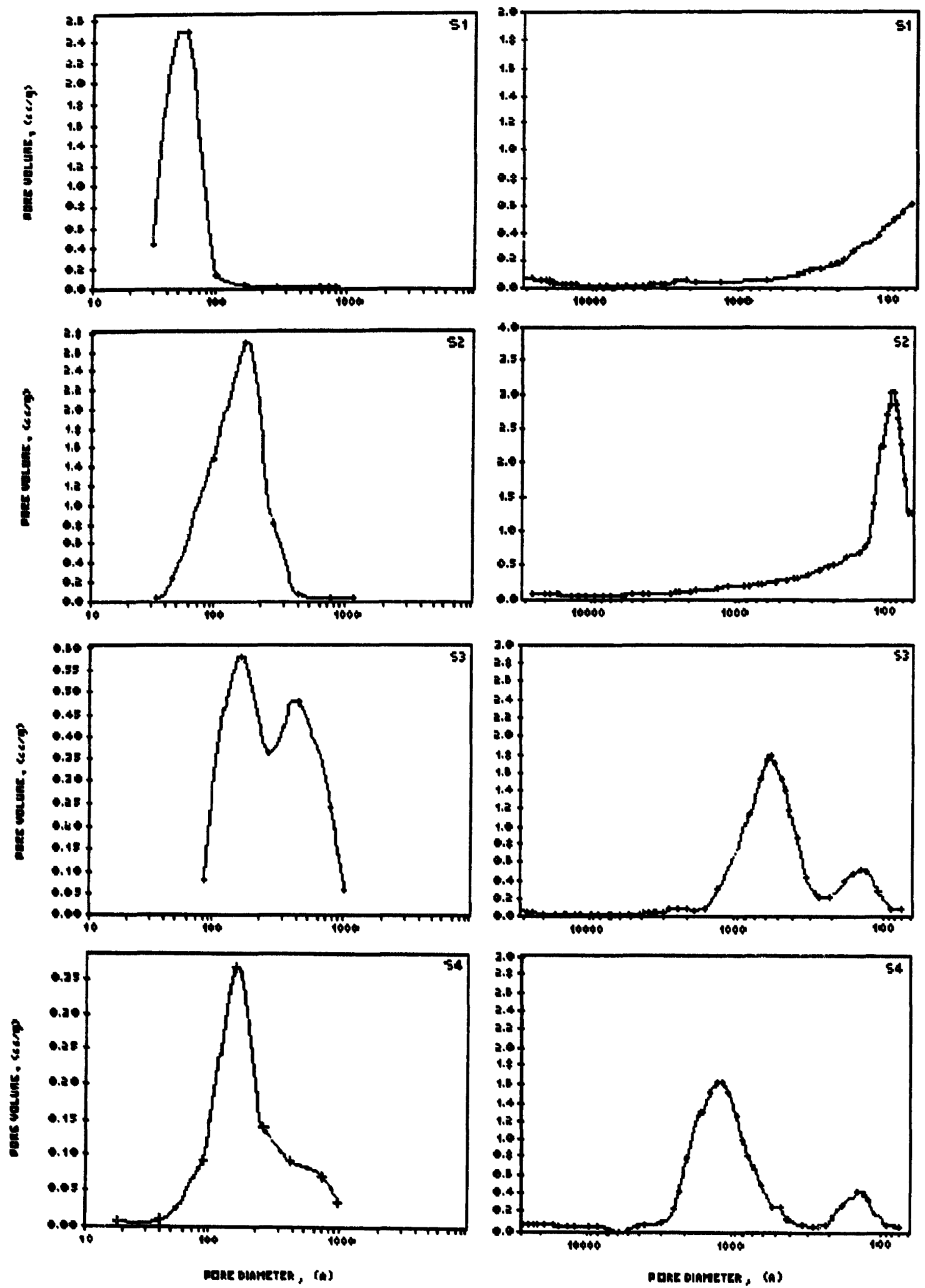

Fïg. 8. Pore sise distributions ( $\mathrm{dV} / \mathrm{dllog}(D))$ of the original silican evaluated from gas adsorption (left), and from mercury porosimetry (right).

\subsection{Water vapour sorption assay}

The results of the water vapour sorption capacity, summarised in Table 3. show that the hydrophilicity of the unmodified silicas decreases according to the following sequence
S1 $>$ S2 $>$ S3 $>$ S4. This trend is obviously related to the surface area of the silicas and. consequently, to the hydroxyl group concentration. The silane derivatives always exhibit higher hydrophobicity when compared to the original silicas, as expected. 


\subsection{Comparison of measured values with manufacturer's specification}

When comparing the results of the surface area, pore diameter and density obtained in this work (Table 3 ) with those specified by the supplier for the original silicas (Table 1), the following can be concluded.

The surface area values of Polygosil 60 and Nucleosil 100 ( samples S1 and S2, respectively), reported in Table 1, are in agreement with those of Table $3\left(450 \mathrm{~m}^{2} \mathrm{~g}^{-1}\right.$ against 468 $\mathrm{m}^{2} \mathrm{~g}^{-1}$ for $\mathrm{S} 1$, and $350 \mathrm{~m}^{2} \mathrm{~g}^{-1}$ against $318 \mathrm{~m}^{2} \mathrm{~g}^{-1}$ for $\mathrm{S} 2$ ). In contrast, for the remaining Nucleosil samples ( $\mathrm{S} 3$ and S4), the deviations between the supplied and the measured values are quite significant $\left(100 \mathrm{~m}^{2} \mathrm{~g}^{-1}\right.$ against $61 \mathrm{~m}^{2} \mathrm{~g}^{-1}$ for $\mathrm{S} 3$. and $25 \mathrm{~m}^{2} \mathrm{~g}^{-1}$ against $48 \mathrm{~m}^{2} \mathrm{~g}^{-1}$ for $\mathrm{S} 4$ ).

As for the mean pore diameters, the results are also somehow confticting. The values measured by gas adsorption for samples $\mathbf{S 1}$ and $\mathbf{S 2}$ are close to those supplied by the manufacturer ( $47 \AA$ against $60 \AA$ for sample $S 1$, and $117 \AA$ against $100 \AA$ for sample S2). Also the value specified in Table 1 for sample S3 $(300 \AA)$ is similar to the corresponding value listed in Table 3 ( $316 \AA$ ). However, it should be noted that the latter was determined by mercury porosimetry, and not by gas adsorption as previously, and that the pore size distribution was bimodal, unlike samples $\mathrm{S} 1$ and $\mathrm{S} 2$. It should be emphasised that, for bimodal distributions, the mean pore diameter does not give a real indication of the central tendency of the distribution and should, therefore, be regarded with caution. With respect to sample $S 4$, which also exhibits a bimodal pore size distribution curve, the mean pore value obtained by mercury intrusion ( $464 \AA$ ) is far smaller than that given by the manufacturer $(1000 \AA)$. Nevertheless, the mode of one of the peaks of the distribution ( $1200 \AA$ ) confirms the presence of pores of about that size.

Comparing the density values listed in Table I with those of Table 3 (true density and bulk density), it is clear that the density specified by the manufacturer is the bulk density, and also that these values are generally comparable to those obtained in this work.

Finally, with regard to particle size, in addition to the manufacturers indication that particles were in the size range 25$40 \mu \mathrm{m}$ for all the samples, it was found that the Polygosil particles are definitely larger than those of the Nucleosil. Furthermore, the particle size distribution curves obtained by laser diffraction show that all the samples have a small amount of very fine particles $(<20 \mu \mathrm{m})$. This may cause unexpected problems such as, for instance, pressure drops higher than those estimated assuming monomodal and narrowly distributed samples.

From this, it is apparent that the specifications given by the supplier are manifestly insufficient and, sometimes, incorrect. Furthermore, since the techniques used for particle characterisation greatly influence the results, it is essential to identify the values reported with the corresponding techniques.

\section{Conclusions}

In the present study, various porous silicas were examined which, based on their commercial designations, were expected to vary in the mean pore size and. consequently, in the surface area. The characterisation carried out in this work provided further information about the pore size distribution, whose knowledge is, for many practical applications, more important than that of surface area or mean pore diameter. It was found that the values supplied by manufacturers did not always coincide with those measured with the techniques used.

Hence, before attempting to correlate or interpret the performance of a given support with a specific reaction, it is strongly recommended that a thorough investigation on the support surface texture should be undertaken, preferably after silanisation. Moreover, due to its relevance, this matter deserves more attention than the one that is generally given by the supports manufacturers.

\section{Acknowledgements}

E.S., G.M. and W.M. gratefully acknowledge the support given to this work by the University of Gent, GOA grant 12053695.

\section{References}

(1) H.H. Weetall, A.M. Filbert, Methods Enzymol. 34 (1974) 59.

[2] D.L. Eaton, in: Leyden. Collins (Eds.). Silylated Surfaces, Vol. 7. Gordon and Brench. Science Publishers. 1980, p. 201.

[3] T.H. Elmer, in: Leyden, Collins (Eds.). Silylated Surfaces, Vol. 7. Gordon and Brench, Science Publishers. 1980, p. 1.

14| C. Horváth, in: Leyden. Collins (Eds.). Silylated Surfaces, Vol. 7. Gordon and Brench. Science Publishers. 1980, p. 269.

[5] G.P. Royer, F.A. Liberatore, in: Leyden, Collins (Eds.). Silylated Surfaces, Vol. 7, Gordon and Brench, Science Publishers, 1980. p. 189.

[6] K. Hashimoto, T. Fujisawa, M. Kobayashi, R. Yosomıya. J. Macromol. Sc1.-Chem. A 18 (2) (1982) 173.

17] E. Stratilová, M. Capka, L. Rexová-Benková, Biocatalysis 2 (1989) 317.

[8] J.A. Bosley, J.C. Clayton. Biotechnol. Bioeng. 43 ( 1994) 934.

[9] D.K. Oladepo, P. Halling. V.F. Larsen. Biocatal. Biotransformation 12 (1995) 47

[10] B.B. Weiner, in: H.G. Barth (Ed. ), Modern Methods of Particle Size Analysis, Wiley, New York. 1984.

1111 S. Brunauer, P.H. Emmett. E. Teller. J. Am. Chem. Soc. 60 (1938) 309.

[12] E.P. Barrett, L.G. Joyner, P.B. Hallenda. J. Am. Chem. Soc 73 ( 1951 ) 373.

[13] E.W. Washburn, Proc. Nat. Acad. Sci. 7 (1921) 115.

114] G. Boven, M.L.C.M. Oosterling, G. Challa. A.J. Schouten, Polymer 31 ( 1990$) 2377$.

[15] British Standard 7591, Porosity and Pore Size Distribution of Materials. Part 2. Method of Evaluation by Gas Adsorption. 1992.

[16] S.J. Gregg, K.S.W. Sing, Adsorption, Surface Area and Porosimetry. 2nd edn., Academic Press, London. 1991.

117] J. Van Brackel, S. Modrý, M. Svatá. Powder Technol. 29 (1981) 1. 